\title{
Prevalence of Restless Leg Syndrome in Pregnancy- A Follow-up Study (PEARLS Study)
}

\author{
1Divya Devaraj, ${ }^{2}$ Uma Devaraj, ${ }^{3}$ Mark Bothello, ${ }^{4}$ Priya Ramachandran, ${ }^{5}$ Uma Maheswari, ${ }^{6}$ George A D'Souza
}

\section{ABSTRACT}

Background: Restless leg syndrome (RLS) is a sensorimotor sleep disorder. The prevalence of RLS during pregnancy is 2 to 3 times more than in the general population.

Objectives: To estimate the prevalence of RLS in pregnancy in Indian population and its association with serum ferritin levels.

Materials and methods: Three hundred twenty-five pregnant women were enrolled. Subjects were grouped as RLS positive (49 subjects) and controls (276) based on the personal interview and were followed 6 months later by telephonic interview.

Results: The subjects' mean age was $25.15 \pm 3.86$ years (range 18 to 38 years). The mean BMI of the subjects was $26.22 \pm 5.31$ $\mathrm{kg} / \mathrm{m}^{2}$. The prevalence of RLS was $15.1 \%$ (49 subjects), 30 of whom were primigravida. Three, 6 and 40 subjects were in their first, second and third trimester of pregnancy respectively.

The distribution of age, body mass index (BMI), diabetes, and hypertension were similar in the two groups. The number of hours of sleep was significantly less in the RLS positive group ( 7.93 vs. 7.5 hours; $p$-value $=0.017$ ). The levels of hemoglobin, oral iron supplements and serum ferritin and baby's birthweight did not differ significantly between the two groups.

Forty-five women reported resolution of RLS symptoms, while two women had persistent RLS symptoms, six months after delivery. Two women were lost to follow-up.

Conclusion: The prevalence of RLS in pregnant Indian women is $15.1 \%$, which was previously undiagnosed. RLS was more prevalent in the third trimester of pregnancy. Neither multiparity or low serum hemoglobin or ferritin were associated with RLS in pregnancy.

Keywords: Brain iron deficiency, Pregnancy, Restless leg syndrome, Serum ferritin.

How to cite this article: Devaraj $D$, Devaraj $U$, Bothello $M$, Ramachandran P, Maheswari U, D'Souza GA. Prevalence of Restless Leg Syndrome in Pregnancy-A Follow-up Study (PEARLS Study). Indian Sleep Med 2018;13(4):57-61.

Source of Support: Each author states that they have not received any grants or funding and not involved in any personal

\footnotetext{
${ }^{1,3}$ Student, ${ }^{2}$ Associate Professor, ${ }^{4}$ Additional Professor, ${ }^{5} \mathrm{HOD}$ and Professor, ${ }^{6}$ Professor and Dean

${ }^{1-6}$ Department of Pulmonary Medicine, St. John's Medical College Hospital, Bengaluru, India
}

Corresponding Author: Uma Devaraj, Department of Pulmonary Medicine, St. John's Medical College Hospital, Bengaluru, India Phone: +9108022065802, e-mail: druma. devaraj@gmail.com relationships, interests, and affiliations over the past three years, that could be perceived as a potential conflict of interest. None of the authors have received any industry related grants or funding, employment, affiliations, patents, inventions, honoraria, consultancies, royalties, stock options/ownership, or expert testimony.

\section{Conflict of interest: None}

Current knowledge/Study rationale: The RLS is an important sleep disorder during pregnancy. There are no data available on RLS in pregnancy from India.

Study impact: The prevalence of RLS in pregnancy in this study was $15.1 \%$, and there was no correlation between the presence of RLS and serum iron studies- such as serum ferritin and hemoglobin levels. Further studies are needed to explore the causation of RLS in pregnancy.

\section{INTRODUCTION}

Restless leg syndrome (RLS) or Willis-Ekbom disease (WED) is a type of sleep disorder that is not very well recognized in clinical practice. RLS is a neurologic condition characterized by an urge to move, associated with or without paresthesia, that occurs or worsens at rest and is relieved by activity. These symptoms should not be secondary to leg cramps, positional discomfort, venous stasis, leg edema, and compression or stretch neuropathies. ${ }^{1}$ To date, all the epidemiological studies have reported the prevalence between $2 \%$ and $15 \%$ in general population. $^{2}$

The RLS results in poor sleep quality and affects the overall quality of life in these patients. Early identification and its treatment would help to prevent morbidity due to RLS.

The RLS has been related to several medical conditions, especially uremia, anemia, pregnancy, and neuropathies. There is substantial evidence for a genetic contribution to RLS. There is increasing evidence for the presence of brain iron deficiency in patients with RLS.

Several epidemiologic studies from Europe, Australia, and China, have shown the prevalence of RLS during pregnancy to be 2-3 times more than in the general population, affecting about 11.2 to $38.8 \%$ of pregnant women. ${ }^{3-8}$ In an extensive study of 16,528 pregnant women living in Japan, the prevalence of RLS was found to be $19.9 \%$.

On conducting a literature search in Pubmed and MedIND, using keywords "restless leg syndrome," 
"pregnancy" and "India," there was no study addressing exclusively, the prevalence of RLS in pregnancy. The prevalence of all sleep disorders in pregnant women in India was reported by Sharma et al. ${ }^{10}$

The trimester and the parity influence the prevalence of RLS in pregnancy. The prevalence of RLS increases with progression of pregnancy. Neyal et al. have described the RLS prevalence as $15.6 \%, 32.8 \%$ and $38.8 \%$ in the first, second and third trimester respectively. ${ }^{6}$

The RLS symptoms typically increase and are maximum in the 3rd trimester and decrease noticeably soon after childbirth. Hormonal changes, iron, and folate status contribute to the pathophysiology of RLS during pregnancy. The prognosis of RLS during pregnancy is better as compared to idiopathic RLS and symptoms are usually relieved for many, by one month after childbirth. ${ }^{11}$

Although the mechanism of development is unknown, there is circumstantial evidence for a role of the dopaminergic system and brain iron status in the pathophysiology of RLS. ${ }^{12}$ Tyrosine hydroxylase is the rate-limiting enzyme in the production of dopamine and requires iron as a cofactor. ${ }^{13}$ Therefore, iron deficiency may affect dopamine production indirectly, while dopaminergic agents are effective in therapy for idiopathic RLS.

This study aims to add strength to the evidence that RLS is probably as prevalent among pregnant women as seen in the above studies. We also aim to study the correlation of severity of RLS with iron deficiency.

\section{Aims and Objectives}

This study aimed to estimate the prevalence of RLS among pregnant women in South India. Characterization of the severity of RLS with IRLS score and correlation of severity of RLS with hemoglobin and biochemical markers such as serum ferritin were the secondary objectives of this study.

The follow-up study was done to examine the resolution of symptoms after the delivery and the effect of RLS on the baby's birth weight.

\section{Sample size}

Assuming the RLS prevalence of $15 \%$ in pregnant women from the previous study of sleep disorders in pregnant women in north India, to estimate a $25 \%$ prevalence of RLS in pregnant ladies, the sample size required to estimate with $80 \%$ power and 5\% alpha error was 205 subjects.

\section{MATERIALS AND METHODS}

This prospective observational study was initiated after institutional ethics committee approval and written informed consent from the subjects.
All consecutive pregnant women aged 18 years or older in the obstetrics outpatient department and inpatient obstetrics unit in a tertiary care center were interviewed using a predesigned pro forma. Subjects with medical conditions associated with RLS such as peripheral neuropathy, medications (sedating antihistamines, dopamine antagonists) were excluded from the study.

The first part of the study (personal interviews) was done from February to August 2016 and the follow-up was completed from September 2016 to February 2017.

Data regarding the demographics, caffeine and tobacco use, smoking history, alcohol use, and comorbid illness were collected. The trimester in which the subject was in, number of pregnancies, other medical diagnoses (obstructive sleep apnea by the Berlin questionnaire, any inflammatory conditions) were also noted.

The RLS diagnosis was made based on revised international RLS study group (IRLSSG) consisting five essential criteria for the diagnosis of RLS. ${ }^{14}$ The RLS symptoms were specifically differentiated from other mimics such as leg cramps, positional discomfort, venous stasis, and leg edema by detailed history and examination. The RLS diagnosis was validated by one of the authors, certified sleep specialists (UD/PR). The family history of any relatives with RLS symptoms was also gathered, as the genetic transmission is known.

The RLS subjects who were diagnosed according to the IRLSSG criteria underwent IRLS severity score questionnaire. Biochemical and lab values such as serum ferritin and hemoglobin was collected at the time of interview. Data collected were analyzed with Statistical Package for the Social Sciences (SPSS) version 17.

\section{Statistics}

Descriptive statistics were used to present the categorical variables.

Comparison between the RLS positive and the negative groups was done using Chi-square test for categorical variables and student t-test for continuous parameters. The association of the peripheral iron status values with RLS was evaluated by regression analysis.

\section{RESULTS}

A total of 325 pregnant women were studied. Three women were excluded from the study. Two subjects had allergic rhinitis and were treated with, and one subject had a congestive cardiac failure. The mean age of the subjects was $25.15 \pm 3.86$ years. Age of the women studied ranged from 18 to 38 years, 93\% being younger than 30 years. The mean BMI of the study population was 26.22 $\pm 5.31 \mathrm{~kg} / \mathrm{m}^{2}$. The mean gestational age was $30.21 \pm 8.4$ weeks (range 8 to 40 weeks). 
RLS was diagnosed in 49 (15.1\%) patients. All the 49 subjects had mild RLS by IRLS severity score (range 2 to 4). Three of the women had one of their family members affected by RLS. The RLS positive women were offered treatment (pregabalin/dopamine agonists), but they refused the same due to safety concerns.

Forty-five women reported complete resolution of RLS symptoms, while 2 women had persistent RLS symptoms, six months after delivery. Two women were lost to follow-up.

The characteristics of patients diagnosed with RLS were compared with those who were negative for RLS symptoms (control group) (Table 1). The distribution of age, BMI, comorbid conditions such as diabetes and hypertension were similar in the two groups. Majority of the women $(262,80.6 \%)$ were home-makers, and 222 were in their third trimester at the time of interview. None of the women interviewed had any history of tobacco use, smoking history or alcohol use.

Low hemoglobin was not correlated with the presence of RLS on conducting a McNemar regression analysis (Table 1). Most Indian women routinely receive daily oral iron supplementation during the pregnancy irrespective of iron status. ${ }^{15}$ All the subjects included in this study were on iron and folate supplements.

The levels of hemoglobin (mean value of $11.5 \pm 1.6$ versus $11.4 \pm 1.3 \mathrm{gm} / \mathrm{dL}$; $\mathrm{p}$-value $=0.73$ ), oral iron supplements (mean value of $69.4 \pm 43.3$ vs. $63.3 \pm 37.4 \mathrm{mg}$ of elemental iron per day; $p$-value $=0.37$ ) and serum ferritin

Table 1: Comparison of characteristics between the RLS positive and negative pregnant women

\begin{tabular}{|c|c|c|c|}
\hline Characteristics & $\begin{array}{l}R L S \\
\text { positive } \\
(N=49) \\
\end{array}$ & $\begin{array}{l}\text { RLS negative } \\
(N=276)\end{array}$ & $p$-value \\
\hline Mean age (years) & $25.63 \pm 4.3$ & $25.07 \pm 3.7$ & 0.34 \\
\hline \multicolumn{4}{|l|}{$\begin{array}{l}\text { Socioeconomic } \\
\text { status }\end{array}$} \\
\hline Upper class & $4(8.1 \%)$ & $0(0 \%)$ & 0.07 \\
\hline Upper middle class & $19(38.77 \%)$ & $119(43.11 \%)$ & \\
\hline Lower middle class & $17(34.69 \%)$ & $72(26.08 \%)$ & \\
\hline Upper lower class & $9(18.36 \%)$ & $67(24.27 \%)$ & \\
\hline Lower Class & $0(0 \%)$ & $0(0 \%)$ & \\
\hline BMI (Mean) & $26.3 \pm 5.3$ & $26.21 \pm 5.3$ & 0.8 \\
\hline $\begin{array}{l}\text { Systolic BP mean } \\
(\mathrm{mm} \text { of } \mathrm{Hg})\end{array}$ & $113.4 \pm 10.8$ & $114.4 \pm 11.9$ & 0.6 \\
\hline $\begin{array}{l}\text { Gestational age } \\
\text { mean (weeks) }\end{array}$ & $30.48 \pm 7.8$ & $30.16 \pm 8.5$ & 0.8 \\
\hline $\begin{array}{l}\text { Elemental iron } \\
\text { supplement } \\
\text { mean }(\mathrm{mg})\end{array}$ & $69.4 \pm 43.5$ & $63.3 \pm 37.0$ & 0.3 \\
\hline $\begin{array}{l}\text { Sleep } \\
\text { mean (hours) }\end{array}$ & $7.55 \pm 1.4$ & $7.93 \pm 1.0$ & 0.03 \\
\hline Hemoglobin & $11.5 \pm 1.6$ & $11.4 \pm 1.3$ & 0.7 \\
\hline Sr. Ferritin (mcg/L) & $37.6 \pm 36.0$ & $36.2 \pm 71.8$ & 0.3 \\
\hline $\begin{array}{l}\text { Birth weight of baby } \\
(\mathrm{kg})\end{array}$ & $2.67 \pm 0.6$ & $2.86 \pm 0.6$ & 0.2 \\
\hline
\end{tabular}

(mean value of $37.6 \pm 36.0 \mathrm{vs} .36 .2 \pm 71.8 \mathrm{mcg} / \mathrm{L}$ ) and baby's birth weight $(2.67 \pm 0.6$ vs. $2.86 \pm 0.6$ kilograms $)$ did not differ significantly between the RLS positive and control groups.

But serum ferritin was lower in the RLS positive group despite the mean iron supplement being higher than in the RLS negative group, though not significant statistically. This raises the possibility that the RLS positive group has an iron deficiency that could lead to a higher prevalence of the syndrome.

\section{DISCUSSION}

The prevalence of RLS in the current study was $15.1 \%$. The prevalence of RLS in the general population in south India has been reported to be $2.1 \%{ }^{16}$

One in five pregnant women are affected with RLS in the western countries. ${ }^{1}$ The prevalence of RLS is less in our pregnant population as compared to $17.8 \%$ and $24 \%$ reported by Vahdat et al. in Iran and Neau et al. in France respectively. ${ }^{17,18}$ They have also reported on high consumption of sleep medication in their subjects, which is on the contrary in our population.

The prevalence of RLS in the current study is comparable to that seen in China (11.2\%), in contrast to that reported from western countries. ${ }^{19}$

A family history of RLS is higher in pregnant subjects with RLS as reported by Balendran et al. and Hennessy et al., but only three of our study subjects who were positive for RLS had such a history. ${ }^{8,20}$

Thirty RLS positive women were primigravida, which is contrary to previous studies showing that the presence of RLS increases with multiparity. ${ }^{21}$

RLS symptoms are more frequent and worsen during the third trimester of pregnancy. Of the 49 subjects with RLS in our study, three were in their first trimester, and 6 were in their second trimester of pregnancy and majority $(40 / 49)$ were in the third trimester of pregnancy.

There is evidence for the presence of anemia and iron deficiency in patients with RLS. ${ }^{22}$

Our study showed no difference in hemoglobin levels between subjects having RLS and the control group. This is similar to the study done by Hubner et al. who refuted anemia as a cause for RLS. ${ }^{23}$

The value of iron levels is probably not relevant as a biomarker, as all pregnant women in India receive mandatory iron and folate supplements from the third month of pregnancy.

There is a basic misunderstanding about the relationship between RLS and peripheral iron status. The peripheral iron status as measured by hemoglobin, serum iron levels, and serum ferritin, is generally normal in RLS patients who do not have iron deficiency anemia. These 
values in most studies are not significantly different from those without RLS. What is more important in the RLS patients is a decreased central nervous system (CNS) iron status as emphasized by Earley et al. ${ }^{24}$ The peripheral iron status generally does not differ significantly between those with and without RLS, even in patients with iron deficiency anemia. ${ }^{25}$

Normal peripheral iron levels do not indicate RLS in this population which is probably related to abnormal brain iron status. This is substantiated by the fact that all of these pregnant women were on iron supplements.

Hemoglobin and serum ferritin values were higher in RLS patients, though not significant, in comparison to the control group in our study. This stresses the fact that peripheral iron measures are of doubtful validity as a biomarker, in patients with RLS.

The RLS results in poor sleep quality and affects the overall quality of life in these patients. Our study is consistent with previous studies reporting reduced sleep time in RLS patients. ${ }^{22}$

There are no previous studies of pregnancy-related RLS reported from south India. The strength of our study lies in its prospective design, a large sample size which adds statistical power to the study.

The limitation of this study is that we could not establish the RLS severity in the subjects with a "suggested immobilization test"(SIT). The RLS diagnosis does not require sleep study or the SIT but is made solely on the five criteria suggested by the IRLSSG. The relationship between RLS and iron levels were not evident in this study. Nonetheless, this study cannot conclude that iron deficiency is not associated with RLS prevalence or severity. Limitations of this study also include the absence of a control group of non-pregnant women of similar age. A much larger population is required and/ or additional phenotyping may be needed (i.e., SIT test, ambulatory leg monitoring for periodic leg movements in sleep) supplement to assessing current genomic risk factors. $^{26}$

\section{CONCLUSION}

The prevalence of RLS in south Indian pregnant women is $15.5 \%$. The RLS was undiagnosed in this population previously. RLS was more prevalent in the third trimester of pregnancy.

Neither multiparity or low serum hemoglobin were associated with RLS in pregnancy. The peripheral iron measures are of questionable validity as a biomarker, in patients with RLS. The presence of RLS did not adversely affect the outcome of pregnancy or the baby's birth weight.

\section{Ethical Compliance Statement}

We confirm that we have read the Journal's position on issues involved in ethical publication and affirm that this work is consistent with those guidelines.

\section{Abbreviations List}

CNS : Central nervous system

IRLS : International restless leg scale

IRLSSG : International RLS study group

$\mathrm{Kg} / \mathrm{m}^{2} \quad$ : Kilogram/meter square

PEARLS : Prevalence of restless leg syndrome in pregnancy study

RLS : Restless leg syndrome

SIT : Suggested immobilization test

UD/PR : Authors: Uma Devaraj/ Priya Ramachandran

WED : Willis-Ekbom disease

\section{REFERENCES}

1. Picchietti DL, Hensley JG, Bainbridge JL, Lee KA, Manconi $\mathrm{M}, \mathrm{McGregor} J \mathrm{~A}$, et al. Consensus clinical practice guidelines for the diagnosis and treatment of restless legs syndrome/ Willis-Ekbom disease during pregnancy and lactation. Sleep Med Rev. 2015 Mar 1;22:64-77.

2. Meir H. Kryger, William C. Dement, Thomas R. Principles and practice of sleep medicine. 5th ed. St. Louis, Missouri: Elsevier Saunders; 2011. pp1723.

3. Neau JP, Marion P, Mathis S, Julian A, Godeneche G, Larrieu $\mathrm{D}$, et al. Restless legs syndrome and pregnancy: follow-up of pregnant women before and after delivery. Eur Neurol. 2010 Dec 23;64(6):361-366.

4. Wesstrom J, Skalkidou A, Manconi M, Fulda S, SundstromPoromaa I. Pre-pregnancy restless legs syndrome (WillisEkbom Disease) is associated with perinatal depression. J Clin Sleep Med. 2014 May 9;10(5):527-533.

5. Prosperetti C, Manconi M. Restless Legs Syndrome/WillisEkbom Disease and Pregnancy. Sleep Med Clin. 2015Sept 1;10(3):323-329.

6. Neyal A, Senel GB, Aslan R, Nalbantoglu M, Acikgoz S, Yilmaz $\mathrm{N}$, et al. A prospective study of Willis-Ekbom disease/restless legs syndrome during and after pregnancy. Sleep Med. 2015 Sep 1;16(9):1036-1040.

7. Ma S, Shang X, Guo Y, Liu G, Yang J, Xue R. Restless legs syndrome and hypertension in Chinese pregnant women. Neurol Sci. 2015 Feb 4;36(6):877-881.

8. Balendran J, Champion D, Jaaniste T, Welsh A. A common sleep disorder in pregnancy: restless legs syndrome and its predictors. Australian and New Zealand Journal of Obstetrics and Gynaecology. 2011 Jun 1;51(3):262-264.

9. Suzuki K, Ohida T, Sone T, Takemura S, Yokoyama E, Miyake $\mathrm{T}$, et al. The prevalence of restless legs syndrome among pregnant women in Japan and the relationship between restless legs syndrome and sleep problems. Sleep. 2003 Sep 1;26(6):673-677.

10. Sharma SK, Nehra A, Sinha S, Soneja M, Sunesh K, Sreenivas $\mathrm{V}$, et al. Sleep disorders in pregnancy and their association with pregnancy outcomes: a prospective observational study. Sleep Breath. 2016 Mar 1;20(1):87-93. 
11. Srivanitchapoom P, Pandey S, Hallett M. Restless legs syndrome and pregnancy: a review. Parkinsonism Relat Disord. $2014 \mathrm{Jul}$;20(7):716-722.

12. Rangarajan S, D'Souza GA. Restless legs syndrome in Indian patients having iron deficiency anemia in a tertiary care hospital. Sleep medicine [Internet]. 2007 Apr 1;8(3):247-251.

13. Cooper JR, Bloom FE, Roth RH. The Biochemical Basis of Neuropharmacology. Oxford University Press. 1991.

14. Allen RP, Picchietti DL, Garcia-Borreguero D, Ondo WG, Walters AS, Winkelman JW, Zucconi M, Ferri R, Trenkwalder C, Lee HB, International Restless Legs Syndrome Study Group. Restless legs syndrome/Willis-Ekbom disease diagnostic criteria: updated International Restless Legs Syndrome Study Group (IRLSSG) consensus criteria-history, rationale, description, and significance. Sleep medicine. 2014 Aug 1;15(8):860-873.

15. Shankar H, Kumar N, Sandhir R, Mittal S, Kurra S, Dhaliwal $\mathrm{L}$, et al. Weekly iron folic acid supplementation plays differential role in maintaining iron markers level in non-anaemic and anaemic primigravida: A randomized controlled study. Saudi Journal of Biological Sciences 2016 Nov 1;23(6):724-730.

16. Rangarajan S, Rangarajan S, D'Souza GA. Restless legs syndrome in an Indian urban population. Sleep Medicine 2007 Dec;9(1):88-93.

17. Vahdat M, Sariri E, Miri S, Rohani M, Kashanian M, Sabet A, et al. Prevalence and associated features of restless legs syndrome in a population of Iranian women during pregnancy. Int J Gynaecol Obstet. 2013 Oct 1;123(1):46-49.

18. Neau JP, Porcheron A, Mathis S, Julian A, Meurice JC, Paquereau J, et al. Restless legs syndrome and pregnancy: a questionnaire study in the Poitiers District, France. Eur Neurol. 2010;64(5):268-274.

19. Shang X, Yang J, Guo Y, Ma S, Jia Z, Xue R. Restless legs syndrome among pregnant women in China: prevalence and risk factors. Sleep Breath. 2014 Dec 2;19(3):1093-1099.

20. Hennessy MD, De La Torre FA. Heredity of restless legs syndrome in a pregnant population. J Obstet Gynecol Neonatal Nurs. 2013 Nov ;42(6):737-748.

21. Sikandar R, Khealani BA, Wasay M. Predictors of restless legs syndrome in pregnancy: a hospital based cross sectional survey from Pakistan. Sleep Med. 2009 Jun 1;10(6):676-678.

22. Lee KA, Zaffke ME, Baratte-Beebe K. Restless legs syndrome and sleep disturbance during pregnancy: the role of folate and iron. J Womens Health Gend Based Med. 2001 May 1;10(4):335341.

23. Hubner A, Krafft A, Gadient S, Werth E, Zimmermann R, Bassetti CL. Characteristics and determinants of restless legs syndrome in pregnancy: a prospective study. Neurology. 2013 Feb 19;80(8):738-742.

24. Earley CJ, Allen RP, Beard JL, Connor JR. Insight into the pathophysiology of restless legs syndrome. Journal of Neuroscience Research [Internet]. 2000 Dec 1;62(5):623-628.

25. Allen RP, Auerbach S, Bahrain H, Auerbach M, Earley CJ. The prevalence and impact of restless legs syndrome on patients with iron deficiency anemia. American journal of hematology [Internet]. 2013 Apr;88(4):261-264.

26. Schormair B, Zhao C, Bell S, Tilch E, Salminen A V, Pütz B, et al. Identification of novel risk loci for restless legs syndrome in genome-wide association studies in individuals of European ancestry: a meta-analysis. The Lancet Neurology [Internet]. 2017 Nov 30;16(11):898-907. 